The researchers said, "For a bulk conductivity constant with nanotube diameter, the greater the number of contact points, the greater will be the resistance change upon exposure to hydrogen. Therefore, the smaller-diameter tubes, with thinner walls and a greater number of contact points, will exhibit higher sensitivities than their larger-diameter counterparts."

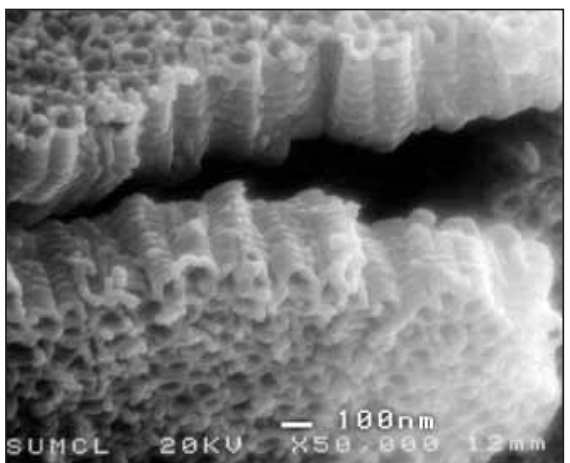

Figure. Vertical cross section of titanium nanotubes used for sensing hydrogen. Credit: Penn State, Craig A. Grimes.
The researchers studied titanium nanotubes of $22 \mathrm{~nm}$ and $76 \mathrm{~nm}$ diameters, which differed in surface area by a factor of two. They found that the response to hydrogen of the smaller tubes is $200 x$ more sensitive than that of the 76-nmdiameter tubes. The researchers suggested that the hydrogen molecules become dissociated at the titanium surface, diffusing into the titania lattice, and act as electron donors, and that this mechanism makes the nanotubes sensitive to hydrogen.

Grimes said results show that titanium nanotube sensors can monitor hydrogen levels from $1 \mathrm{ppm}$ to $4 \%$. Furthermore, the material is not "used up" when sensing hydrogen, but once the gas clears from the tubes, can be used again. The researchers tested the titanium nanotubes with carbon dioxide, carbon monoxide, ammonia, and oxygen, finding little interference.

"Many researchers have tried to use carbon nanotubes as gas sensors, but they do not work very well," said Grimes. "Titania has really great sensitivity and a nice response."

\section{Highly Ordered Merocyanine Dye Assemblies Reminiscent of Chlorophyll Dye Rods}

Photosynthesis relies on highly organized dye assemblies for electron transfer. An orderly arrangement of dyes is also thought to be critical for organic materials in electronic and photonic applications. Typically, however, stacking of $\pi$-bonded systems results in one-dimensional dye assemblies, although two-dimensional brickwork and tubular aggregates of cyanine dyes have been discovered serendipitously. F. Würthner and S. Yao of Universität Würzburg, Germany, and U. Beginn of RWTH Aachen, Germany, have recently applied concepts of supramolecular polymerization and hierarchical selforganization to fabricate, in a controlled manner, highly ordered merocyanine dye assemblies.

As reported in the July 21 issue of Angewandte Chemie, Würthner and coworkers attached nonpolar tridodecyloxybenzyl substituents to highly polar merocyanine and bis(merocyanine) dyes, which made them soluble in low-polarity

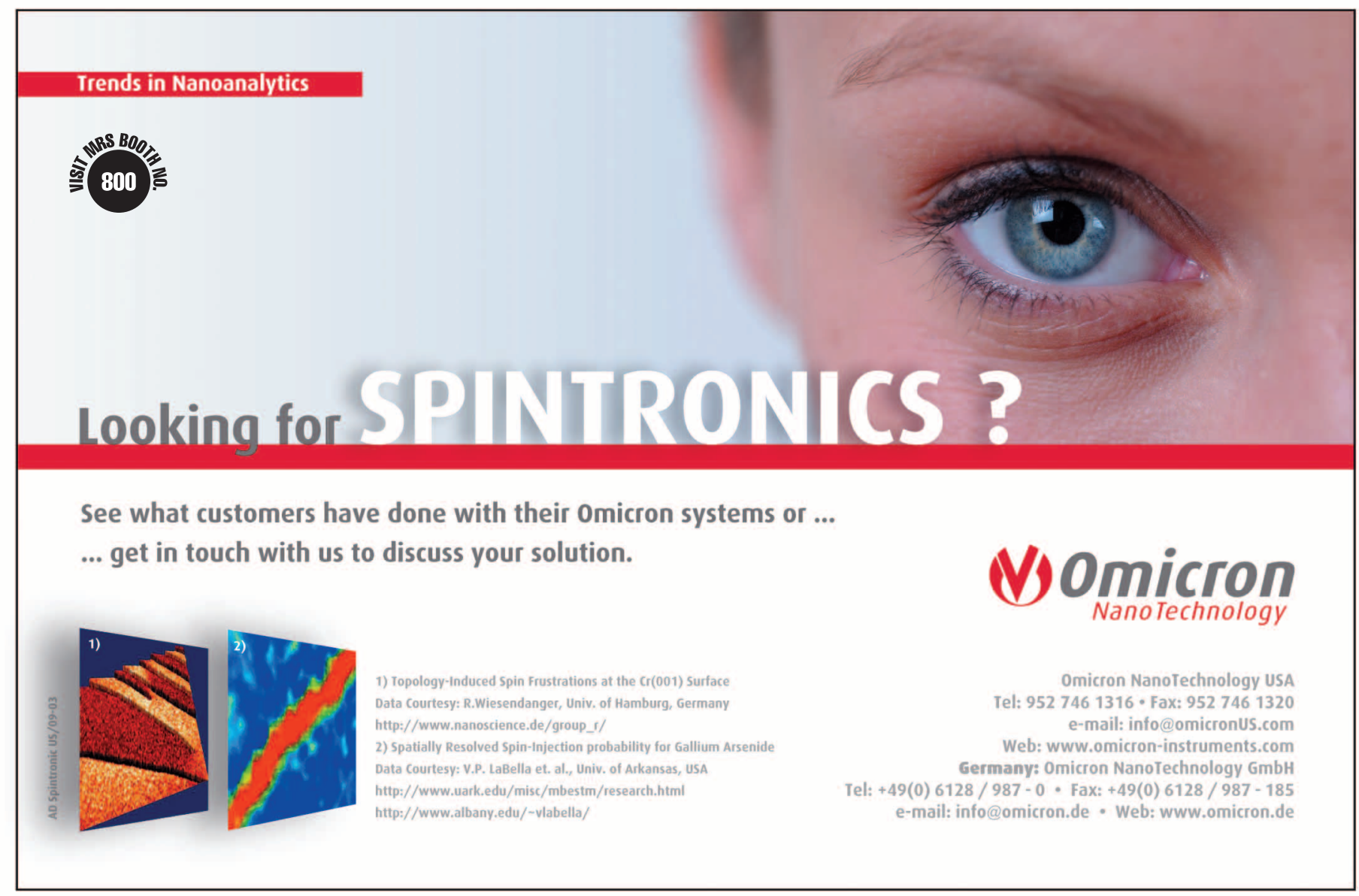

For more information, see http://advertisers.mrs.org 
solvents. The researchers had previously shown that the extremely high dipole moment of merocyanine results in large dimerization constants $\left(K_{\mathrm{dim}}>10^{6} \mathrm{M}^{-1}\right)$ in low-polarity solvents. In addition, the chromophore's highly polarizable $\pi$ system provides functionality.

While the substituted merocyanine dimers were shown by UV-vis absorption spectra to be stable in low-polarity solvents, even at low dilution levels, the substituted bis(merocyanine) dyes displayed absorption bands associated with three distinct species. In addition to the monomer band, two blue-shifted bands were observed, that is, their blue shift depended on the solvent. The researchers reported that observation of the less blue-shifted band indicated supramolecular polymerization by way of the two receptor sites in each molecule. Furthermore, observation of the bands that were more blue-shifted suggested to the researchers a further aggregation of the polymeric strands, which formed a more stable assembly. As dye concentration increased, a lyotropic mesophase formed, followed by a solventfree film with an unusual liquid-crystalline texture. X-ray diffraction of the film revealed hexagonal columnar order with a lattice constant of $4.34 \mathrm{~nm}$, which was in good agreement with rod size determined in a transmission electron microscope.

The researchers conclude that a hierarchical growth process occurs in the structures. As solvent polarity is reduced, each of the two chromophores in the bis(merocyanine) dye dimerizes with another dye in an antiparallel manner. The resulting randomly coiled fibrils fold into helical conformations. Six helices then intertwine to form a densely packed rod with the tridodecyloxybenzyl substituents on the outer, solvent-accessible surface, which is favorable in low-polarity solvents. At higher concentrations, interdigitation of the alkyl substituents leads to a decrease in the rod diameter as mesophases and gels are formed. The researchers said that the tubular structure of their dye aggregates is similar to chlorophyl dye rod elements in the chlorosomes of photosynthetic bacteria. In addition, they envision "fascinating possibilities [that] might arise for merocyanine dye assemblies, especially if their intermolecular arrangement could be changed."

STEVEN TROHALAKI

\section{Functionalizable, Biodegradable, and Protein-Resistant Polymer Fabricated}

Protein-resistant materials have wide applications in tissue regeneration, drug delivery, and systems for both patterned cell cultures and high-throughout screening of proteins or cells. Poly(ethylene gly$\mathrm{col})$ (PEG) as a main chain polyether has been shown to be a good candidate, except for its functionalization limits and nonbiodegradability. Also, the involvement of carbohydrate moieties in self-assembled monolayers and polymeric materials has shown superior protein resistance. Combining these two merits, Z. Guan, M. Metzke, and J.Z. Bai of the University of California-Irvine designed a sidechain polyether constructed from the carbohydrate-derived monomers. In addition, this polymer can be degraded by hydrolysis of the ester linkage between the repeating units. The researchers reported their work in the July 2 issue of the Journal of the American Chemical Society.

During preparation, the diol monomer was made from a commercially available reduced sugar. Its primary hydroxyl groups were protected with a trityl group, and its secondary hydroxyl groups were permethylated with iodomethane under a basic condition. Another diacid chloride monomer was then obtained from the diol

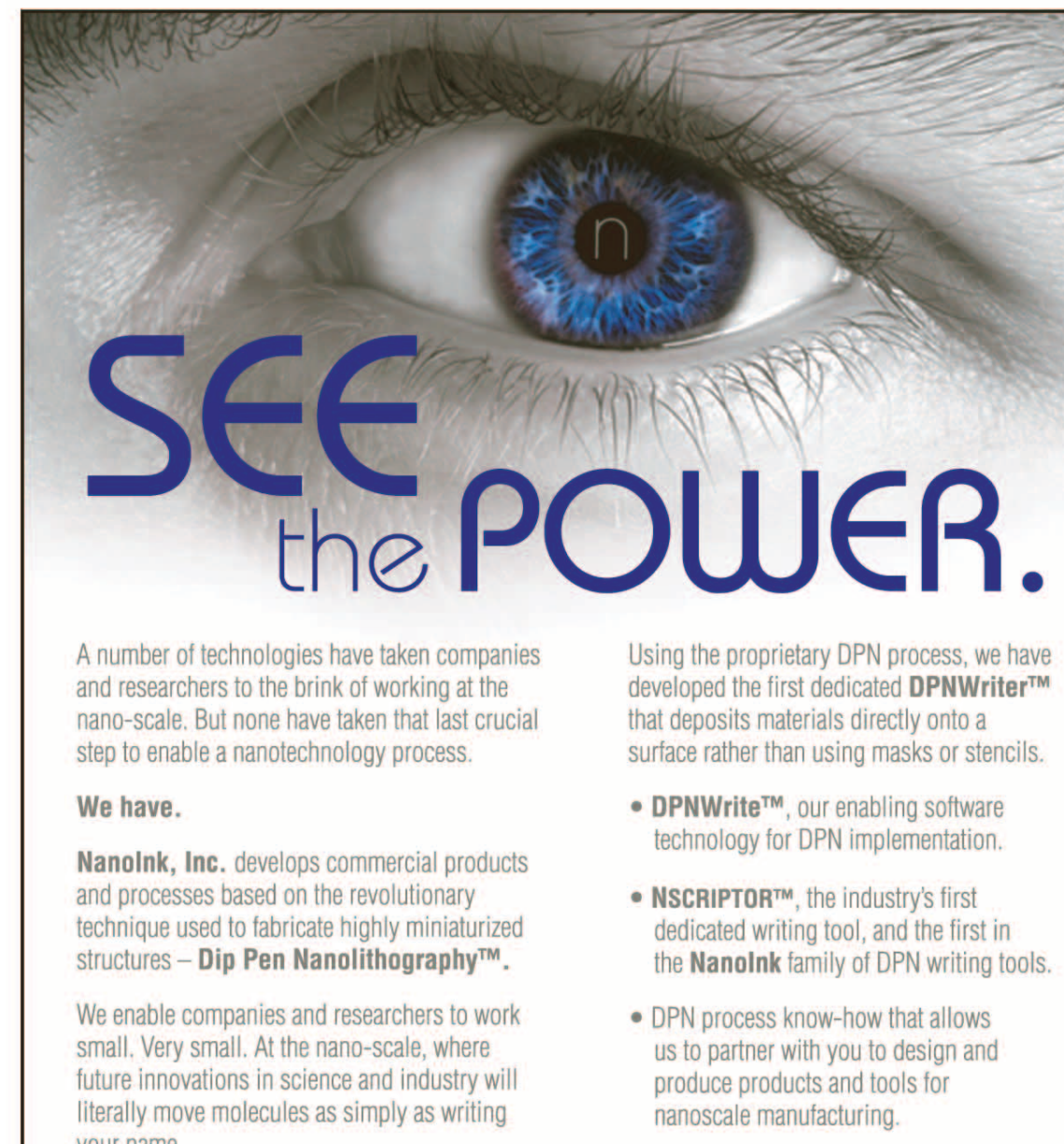

You can see the power of DPN technology today. At Nanolnk.

DPN ${ }^{\mathrm{TM}}$ implements bottom-up nanotechnology that enables direct-write patterning with a truly scalable, repeatable and parallel process.

\section{nAROINK}

1335 West Randolph Street, Chicago, Illinois 60607 www.nanoink.net | 312-525-2900 | sales@nanoink.net

For more information, see http://advertisers.mrs.org 